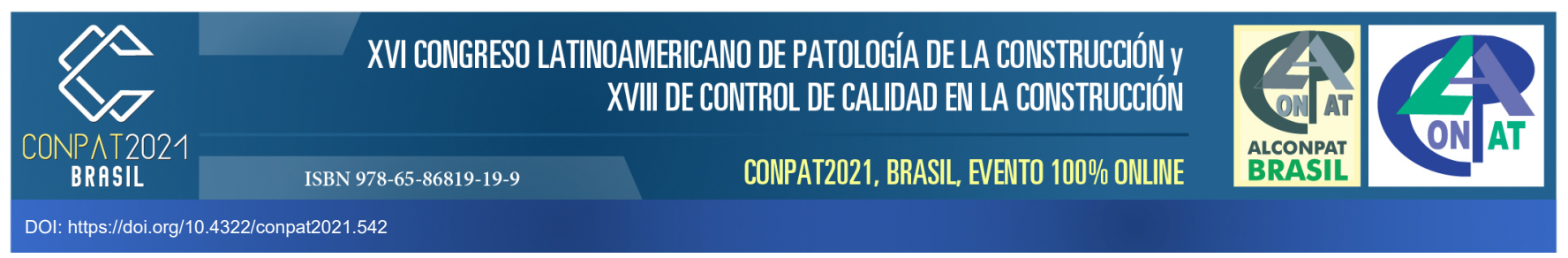

\title{
Análise de vibrações em estruturas de Cross Laminated Timber e concreto
}

\author{
SIQUEIRA, J. M. S. ${ }^{1 *}$, MASCIA, NILSON T. ${ }^{2}$, VILELA, R. ${ }^{3}$ \\ *Autor de Contacto: 191366@dac.unicamp.br \\ ${ }^{1}$ Departamento de Engenharia Civil, Universidade Estadual de Campinas (Unicamp), Campinas Brasil
}

\begin{abstract}
RESUMO
Este trabalho tem como objetivo analisar a contribuição da parcela de concreto no comportamento dinâmico de peças estruturais em CLT-CONCRETO, para serem utilizadas em lajes de pisos. A partir de resultados numéricos, utilizando-se os softwares RFEM e ANSYS, diferentes modos de vibração foram analisados para atender os limites aceitáveis de vibrações estabelecidos pela NBR 7190/1997. Contudo foi obtida uma redução no pico da amplitude do modo de vibrar relacionado à primeira frequência natural de $17,88 \mathrm{~Hz}$ na estrutura de CLT-CONCRETO de $0,8 \%$, se comparada com a mesma estrutura, mas sem a contribuição da parcela de concreto. Verificou-se a partir da análise que a parcela de concreto tem uma contribuição significativa para o aumento da rigidez e também para a frequência natural da estrutura.

Palavras-chave: CLT-CONCRETO; ANÁLISE DINÂMICA; VIBRAÇÕES.
\end{abstract}




\title{
Vibration Analysis in Cross Laminated Timber and Concrete Structures
}

\begin{abstract}
For a better understanding of the mechanical behavior of timber-concrete composite systems, it is considered in this work pieces with composite section of Cross Laminated Timber (CLT) and concrete. This article aims to analyze the dynamic behavior of structural pieces of CLTCONCRETO from the numerical results, using the software RFEM and ANSYS to evaluate the contribution of the concrete part of CLT-concrete pieces to be used as floor slabs.

Different vibration modes are analyzed to obtain relationships, through the natural frequency, that meet the acceptable limits of vibrations established by NBR 7190/1997.

It was verified from the analysis that the concrete part provides significant contribution to the increase of the rigidity and also the natural frequency of the structure.
\end{abstract}

Keywords: CLT-CONCRETO; dynamic analysis; vibrations.

\section{Análisis de vibraciones en estructuras de hormigón y madera laminada cruzada}

\section{RESUMEN}

El presente trabajo tiene como objetivo analizar el aporte de la parcela de hormigón y el comportamiento dinámico de las piezas estructurales en CLT-CONCRETE, para ser utilizadas en forjados. A partir de resultados numéricos, utilizando el software RFEM y ANSYS, se analizaron diferentes modos de vibración para cumplir con los límites de vibración aceptables establecidos por NBR 7190/1997. Sin embargo, se obtuvo una reducción en la amplitud pico del modo de vibración relacionada con la primera frecuencia natural de $17,88 \mathrm{~Hz}$, en la estructura CLTCONCRETE de $0,8 \%$, respecto a la misma estructura, pero sin el aporte de la porción de hormigón. A partir del análisis se verificó que la parcela de hormigón tiene una contribución significativa al aumento de rigidez y a la frecuencia natural de la estructura.

Palabras clave: CLT-CONCRETE; ANÁLISIS DINÁMICO; VIBRACIONES. 


\section{INTRODUCÃO}

O Cross Laminated Timber (CLT) é um material engenheirado de construção usado principalmente na Europa onde foi desenvolvido na década de 1990. Leve, resistente e ecológico, o CLT se tornou um dos materiais mais empregados em métodos construtivos em madeira do mundo (Pereira e Calil, 2019).

Também conhecido como Madeira Lamelada Colada Cruzada (MLCC) no Brasil, o CLT é um material industrializado composto por lamelas de madeira coladas e dispostas em camadas ortogonais entre si. Apesar de poder ser produzido com diversas espécies de madeira, atualmente a sua manufatura tem sido a partir de variedades de pinus e eucalipto, que no Brasil são espécies de madeira de reflorestamento.

Com base neste questionamento, este trabalho visa: avaliar o comportamento dinâmico em placas de CLT-CONCRETO, avaliar suas vulnerabilidades a movimentos de vibração, que muitas vezes geram desconforto aos usuários, causando sensações de insegurança, esses problemas estão relacionados com os estados limites de serviço, percepção da vibração ou incômodo, esses parâmetros visam garantir segurança aos usuários, buscando com isto uma conscientização do uso correto desses materiais e um melhor aproveitamento dos recursos disponíveis para a confecção de pisos, lajes e paredes das edificações que utilizam esses tipos de materiais denominados de compósitos.

Uma peça composta de CLT e concreto tal como mostrado na Figura 1 possui uma tecnologia com otimização de desempenho e qualificação do material, criando uma conexão estrutural entre componentes de madeira e concreto de excelente desempenho estrutural (Clouston e Schreyer, 2006).

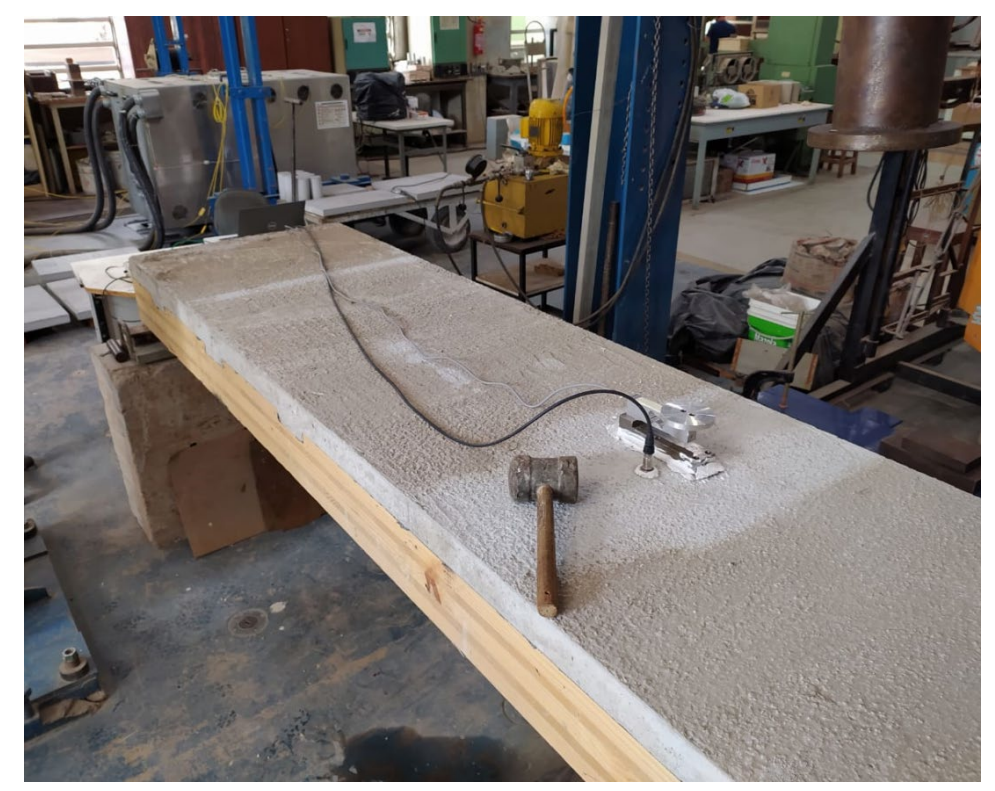

Figura 1. Peça composta de CLT -CONCRETO.

Focando-se em ações dinâmicas nas estruturas que são forças variáveis no tempo, seja em magnitude, sentido ou direção, cujas variações induzem as estruturas ficarem sujeitas as acelerações, velocidades e deslocamentos, gerando forças de inercia e amortecimento, (De Silva, 2000).

Segundo (Lima et al., 2008), “as vibrações podem ser entendidas como movimentos oscilatórios de um sistema em torno de um valor de referência, a sua deformada elástica, a amplitude do movimento oscilatório, determina a magnitude da vibração e sua taxa de repetição, determinando a frequência que o sistema vibra."

Para se avaliar a solicitação dos carregamentos de cargas dinâmicas na estrutura, adota-se uma 
estimativa dos parâmetros modais de edifícios CLT para se compreender a resposta sob condições operacionais de testes in loco, e compreender a lacuna entre a resposta dinâmica possivelmente linear do comportamento da carga de serviço, e o comportamento não linear sob excitação de cargas horizontais (ventos fortes e atividades humanas). A diferença é muito maior para edifícios CLT do que para aqueles em concreto ou aço, uma vez que a resistência do rolamento a cargas laterais estão concentradas nas conexões (Aloisio et al., 2020).

O problema se agrava com a utilização de espécies de madeiras com baixos módulos de elasticidade, propriedade essa que correlaciona a deformação elástica com a tensão, isso pode tornar os pisos suscetíveis à elasticidade e vibração (Jiang e Crocetti, 2019).

Nos diversos casos em que as estruturas apresentam vibrações excessivas com atividades humanas o efeito dinâmico é subestimado, principalmente nas ações dinâmicas moderadas que muitas vezes são tratadas como ações estáticas, em grande parte dos problemas de vibração à média da taxa de deformação $\dot{\varepsilon}=0,1 s^{-1}$ de forma que mudanças nas propriedades dos materiais são apenas moderadas. Um efeito maior ocorre quando $\dot{\varepsilon}$ varia de 1 a $10 s^{-1}$ típico no caso de forças de impacto (Bachmann e Ammann, 1987).

Segundo (Rao, 2008) "as vibrações podem ser entendidas como movimentos periódicos que se repetem após um intervalo de tempo e compreendem a transferência alternada de energia potencial para energia cinética, e de energia cinética para potencial." As fontes de vibração podem ser naturais, causadas por fenômenos da natureza como ventos, terremotos ou causadas pelas atividades humanas, oriundas das movimentações de pessoas, máquinas e equipamentos industriais, tráfego e atividades da construção civil, entre outras.

Um dos pontos de partida mais aceitos em relação à ação humana corresponde à proposta de (Suárez et al., 2019), pressupõe que para frequências naturais superiores a $8 \mathrm{~Hz}$, o componente de baixa frequência do caminhar humano, tende a produzir movimentos que podem ser considerados semiestáticos. O controle da deflexão estática portanto, torna-se um fator decisivo, por outro lado, o impacto do passo excita componentes de frequência mais alta, e a resposta é determinada pela rigidez, massa e razão de amortecimento.

O entendimento das formas modais e de como a estrutura vibrará quando excitada, ajudará o engenheiro projetar melhor a estrutura para aplicações de vibração e ruído. Assim, a análise modal é uma ferramenta poderosa de auxílio ao projeto de estruturas civis.

Conforme (Xing e Liu, 2009) na determinação de soluções exatas para vibração livre de placas, a derivação da expressão matemática do modo de vibração, que satisfaz as equações de problema de autovalor, é a questão mais difícil de ser resolvida, para qual tem dedicado os maiores esforços na tentativa de alcançar soluções aproximadas com grande precisão.

Métodos analíticos muitas vezes derivam de investigações experimentais em um número limitado de tipologias estruturais, uma extensão direta dessas abordagens para diferentes tipos de pisos, deve, portanto, ser verificada. Com todas essas informações pode-se analisar o material com relação à sua fragilidade, quando submetido a condições extremas podendo ser observado seu comportamento nas diferentes situações.

Para se obter uma avaliação mais refinada, métodos simplificados poderiam ser substituídos por métodos mais avançados, tais como uma modelagem numérica capaz de simular melhor as reais propriedades dinâmicas da estrutura nos elementos estruturais e os efeitos de forças externas.

Para verificar o Estado Limite de Serviço (ELS) das vibrações em elementos estruturais de madeira, em ambientes, nos quais há grande movimentação de pessoas, o item 9.3 da NBR 7190 (ABNT, 1997) dispõe que seja adotada uma frequência natural do elemento, superiores a $8 \mathrm{~Hz}$. Por essa razão, a verificação na vibração natural torna-se indispensável em elementos de CLT, para garantir que esses elementos não venham a falhar por vibração excessiva. 


\section{PROCEDIMENTO}

Um modelo numérico utilizado para se analisar o comportamento dinâmico de peças estruturais em CLT-CONCRETO foi desenvolvido com a utilização de dois softwares, usando a pesquisa acadêmica do Ansys Workbench V.19.2. Essa análise numérica visa avaliar a capacidade do modelo de prever o comportamento real das placas de CLT-CONCRETO e no RFEM, sendo esse um software comercial desenvolvido pela Dlubal Software GmbH e se utiliza do (MEF) método de elementos finitos para realizar análises e dimensionamentos estruturais.

Já a análise numérica foi desenvolvida com auxílio do módulo RF-LAMINATE (DLUBAL SOFTWARE GMBH, 2016) do software comercial RFEM 5 (DLUBAL SOFTWARE GMBH, 2013).

Por fim para se comparar e verificar resultados, foi realizado uma simulação de uma peça somente em CLT sem a contribuição da parcela de concreto.

\subsection{Análise Numérica Ansys}

$\mathrm{Na}$ análise numérica adotou-se um modelo 3D FEM (Método de Elementos Finitos) via ANSYS Composite PrepPost. Esse modulo destina-se a determinação de seções transversais para serem aplicadas a elementos de placa. Tais seções são construídas com camadas de materiais sobrepostas, podendo ser materiais ortotrópicos, com a rotação de suas respectivas camadas, como mostra a Figura 2. No software, após a elaboração de cada camada a qual foi atribuída o material e direção principal, o programa cria uma matriz de rigidez para compatibilização dos deslocamentos com os esforços atuantes, para cada elemento finito discretizado no modelo.

O elemento de shell é desenvolvido em Parametric Design Language (APDL) e utilizado para facilitar a geração de várias camadas das lâminas de CLT, com parâmetros de entrada variados. APDL é um script de linguagem, que permite a automação de tarefas comuns, e a criação de modelos com parâmetros definidos pelo usuário. Os comandos APDL abrangem uma ampla gama de recursos.
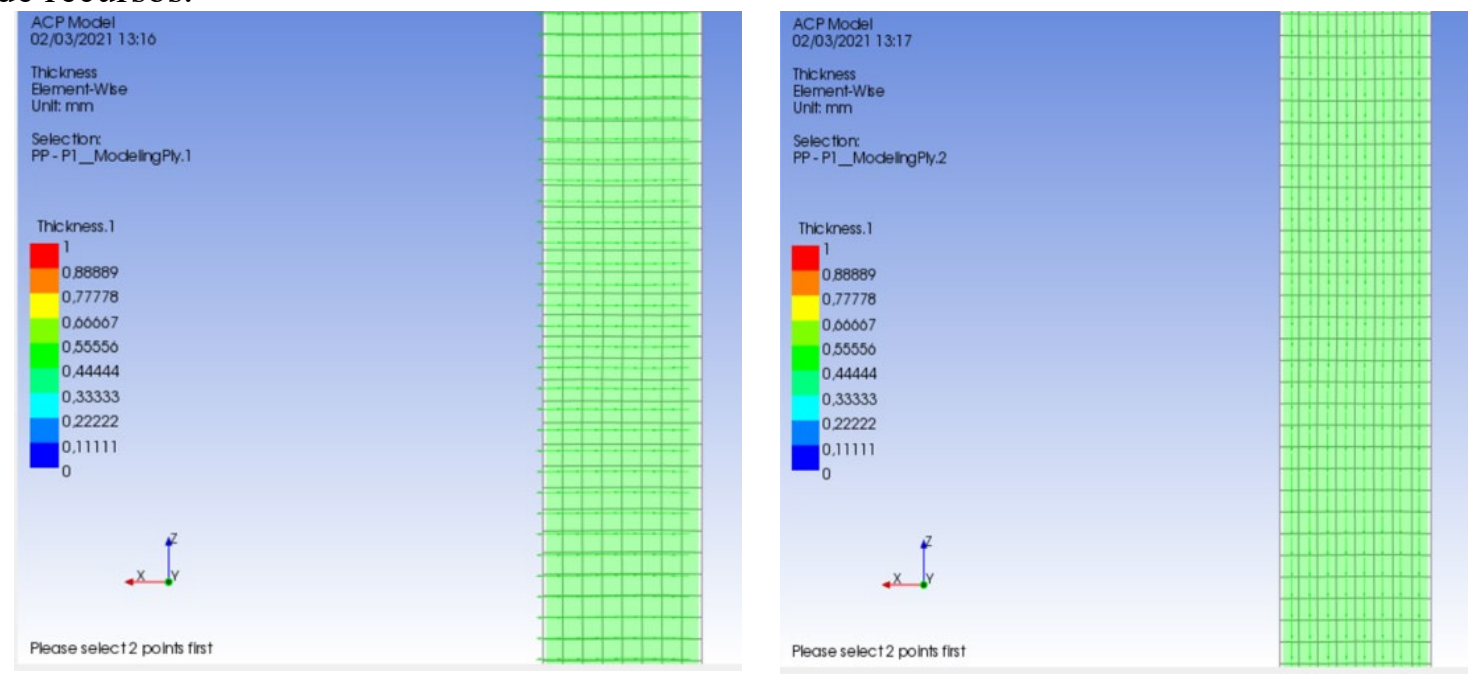

Figura 2. Exemplo de criação de uma seção composta no módulo ACP. 


\subsubsection{Definição da geometría}

Para a simulação numérica do comportamento da placa de CLT-CONCRETO, elaborou-se um modelo de geometria, conforme ilustrado pela Figura 3.

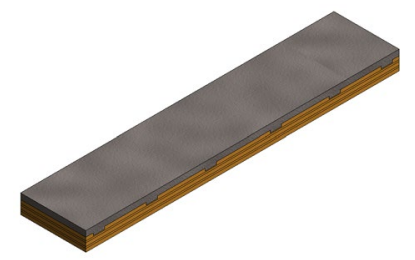

(a) Vista isométrica

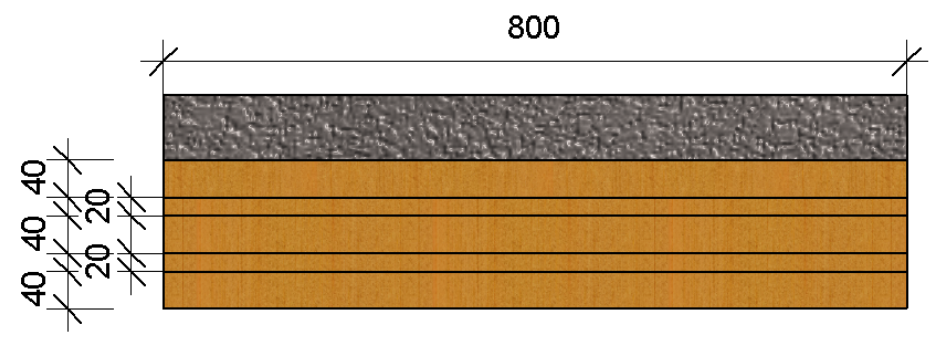

(b) Seção transversal

3840

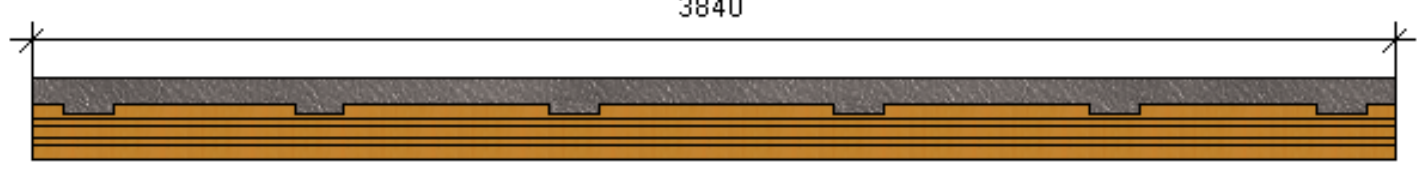

(c) Vista Lateral

Figura 3. Placa com geometria dos ensaios simulada estática e numericamente com o ANSYS.(Dimensões em mm)

Para as propriedades físicas e mecânicas, utilizadas para a simulação numérica dos ensaios experimentais das placas de CLT-CONCRETO, utilizou-se os parâmetros estabelecidos na norma de classificação dos materiais NBR 7190 (ABNT ,1997) para o Pinus taeda, apresentados na Tabela 1. As correlações das propriedades mecânicas da madeira com o modulo de elasticidade longitudinal, considerando-se as direções: radiais, tangenciais e seus respectivos coeficientes de Poisson, foram determinadas com as equações de correlação, (Bodig e Jayne ,1982) apresentadas na Tabela 2. Foi considerado para a análise numérica o concreto da classe $\mathrm{C} 30$.

Tabela 1. Propriedades mecânicas do Pinus taeda

\begin{tabular}{|c|c|}
\hline Propriedades & NBR 7190:1997 \\
\hline$E_{z}(\mathrm{MPa})$ & 13304 \\
\hline$\rho\left(\mathrm{kg} / \mathrm{m}^{3}\right)$ & \\
\hline
\end{tabular}

Tabela 2. Equações para as relações elásticas em função do módulo de elasticidade longitudinal.

\begin{tabular}{|c|c|}
\hline Equações $(\mathrm{GPa} / \mathrm{GPa})$ & $\mathbf{R}^{\mathbf{2}}$ \\
\hline$E_{L} / E_{T}=-0,0174{E_{L}}^{2}+0,9927 E_{L}+11,103$ & 0,9997 \\
\hline$E_{L} / E_{R}=-0,136{E_{L}}^{2}+0,9886 E_{L}+3,0643$ & 0,9990 \\
\hline$E_{L} / G_{L R}=-0,0236 E_{L}{ }^{2}+1,1473 E_{L}+1,1956$ & 0,9999 \\
\hline$G_{L R} / G_{R T}=0,0248 E_{L}{ }^{2}+0,08665 E_{L}+16,811$ & 0,9938 \\
\hline$E_{L} / E_{R}=0,00002 E_{L}{ }^{2}+0,0012 E_{L}+1,0544$ & 0,9951 \\
\hline
\end{tabular}


Tabela 3. Média dos coeficientes de Poisson

\begin{tabular}{|c|c|}
\hline Coeficentes de Poisson & Pinus \\
\hline$v_{L R}$ & $25,96 \cdot 10^{-6} E_{L}$ \\
\hline$v_{R L}$ & $34,95 \cdot 10^{-6} E_{R}$ \\
\hline$v_{L T}$ & $31,04 \cdot 10^{-6} E_{L}$ \\
\hline$v_{T L}$ & $25,96 \cdot 10^{-6} E_{T}$ \\
\hline$v_{R T}$ & $539,98 \cdot 10^{-6} E_{R}$ \\
\hline$v_{T R}$ & $620,04 \cdot 10^{-6} E_{T}$ \\
\hline
\end{tabular}

Tabela 4. Correlação das propriedades mecânicas utilizadas no modelo numérico

\begin{tabular}{|c|c|}
\hline Propriedades mecânicas & Bodig e Jayne (1982) \\
\hline$E_{z}(M P a)$ & 13304 \\
\hline$E_{y}(M P a)$ & 696 \\
\hline$E_{x}(M P a)$ & 621 \\
\hline$v_{z y}$ & 0,35 \\
\hline$v_{x y}$ & 0,52 \\
\hline$v_{z x}$ & 0,41 \\
\hline$G_{z y}(M P a)$ & 799 \\
\hline$G_{x y}(M P a)$ & 82 \\
\hline$G_{z x}(M P a)$ & 799 \\
\hline
\end{tabular}

\subsubsection{Elementos de Malha Ansys}

O elemento de malha SOLID185 foi usado para modelagem 3-D de estruturas sólidas, sendo definido por oito nós, com três graus de liberdade em cada nó, com translações nodais nas direções x, y e z. A localização do nó para este elemento é mostrada na Figura 4.

O elemento SOLID185 de Geometria Estrutural Homogênea Sólida é definido por oito nós com propriedades de material ortotrópico. O sistema de coordenadas de elementos padrão está localizado ao longo das direções globais, consoante com as direções de material ortotrópico.

O modelo possui 35805 nós e 29568 elementos, sendo analisada a discretização da malha com as dimensões máximas de $5 \mathrm{~cm}$. Para cada elemento finito utilizado como referência foram considerados até o oitavo modo de vibração.

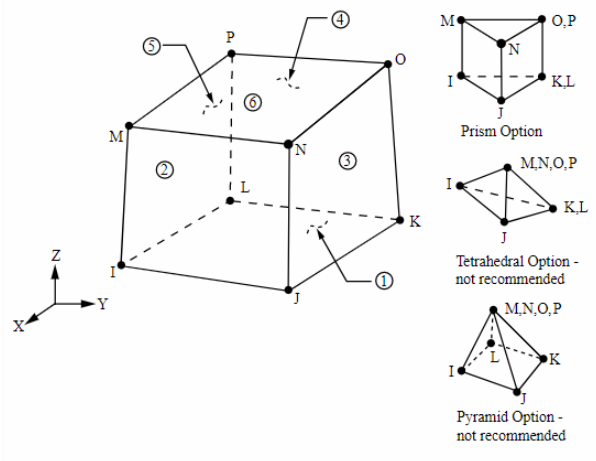

Figura 4. Solid 185 Geometria estrutural 
Na Figura 5 apresenta a discretização do modelo numérico.

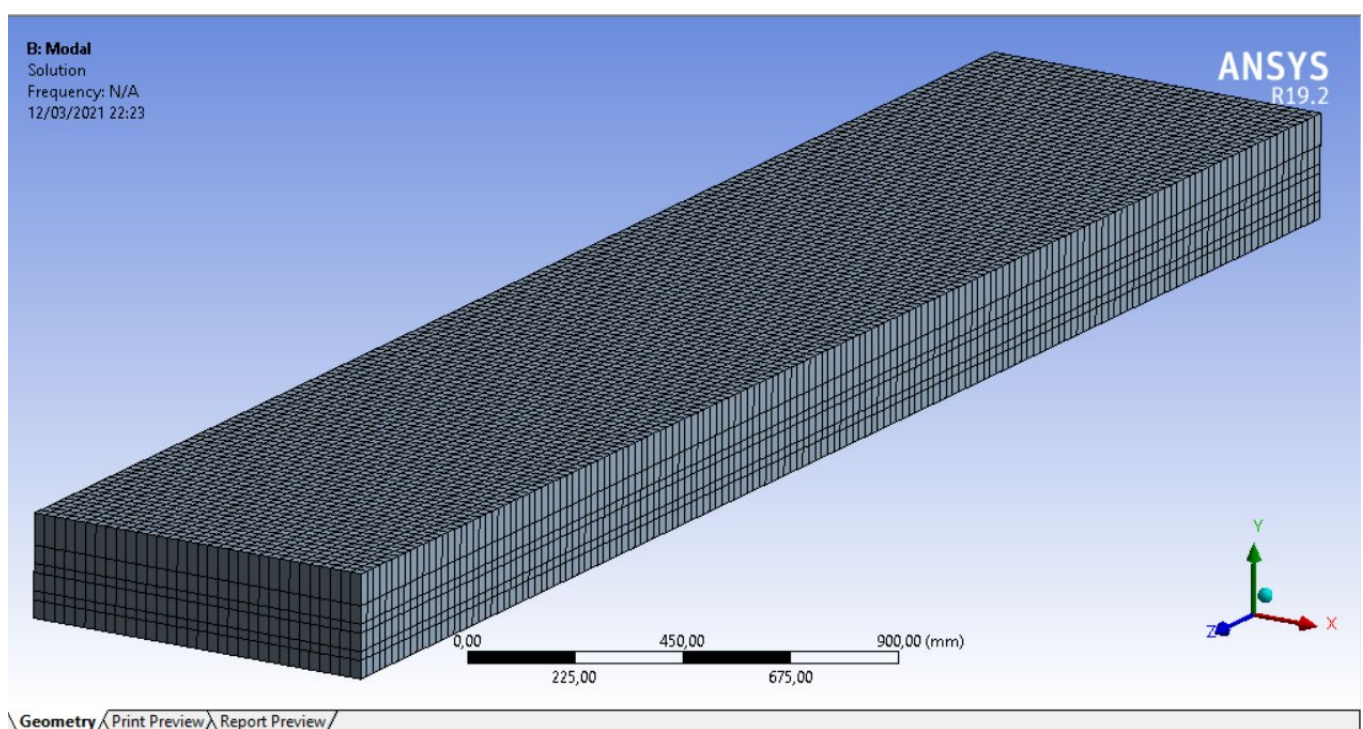

Figura 5. Discretização do modelo numérico

Observa-se que com a mesma geometria foi desenvolvido um modelo numérico para uso do software RFEM.

\subsection{ANÁLISE NUMERICA RFEM}

Inicialmente a geometria do sistema estrutural é solicitada pelo programa RFEM. Essa pode ser definida em função do tipo de estrutura a ser analisada. As dimensões da placa foram limitadas por pontos, que foram conectados por linhas, seguidamente, preenchidos com elementos de placa. Não foram impostas condições de contorno subsidiárias de simetria no modelo. O RF-LAMINATE destina-se a determinação de seções transversais a fim de serem aplicadas a elementos de placa. Tais seções são construídas com camadas de materiais sobrepostas, podendo ser de materiais ortotrópicos. Por essa razão, tal módulo é destinado para modelos de compósitos, pois pode-se utilizar as propriedades mecânicas de materiais ortotrópicos com suas respectivas camadas, conforme mostra a Figura 6.

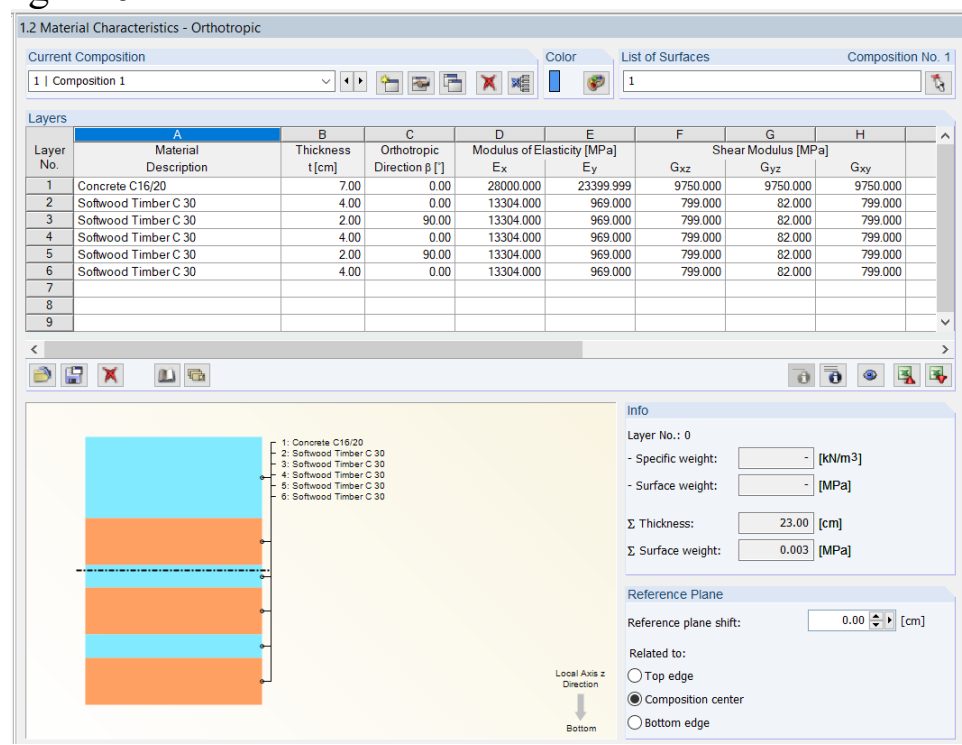

Figura 6. Exemplo de criação de uma seção composta no módulo RF-LAMINATE 
Na Figura 7 são apresentadas as dimensões longitudinais e transversais utilizadas para o desenvolvimento do modelo numérico.

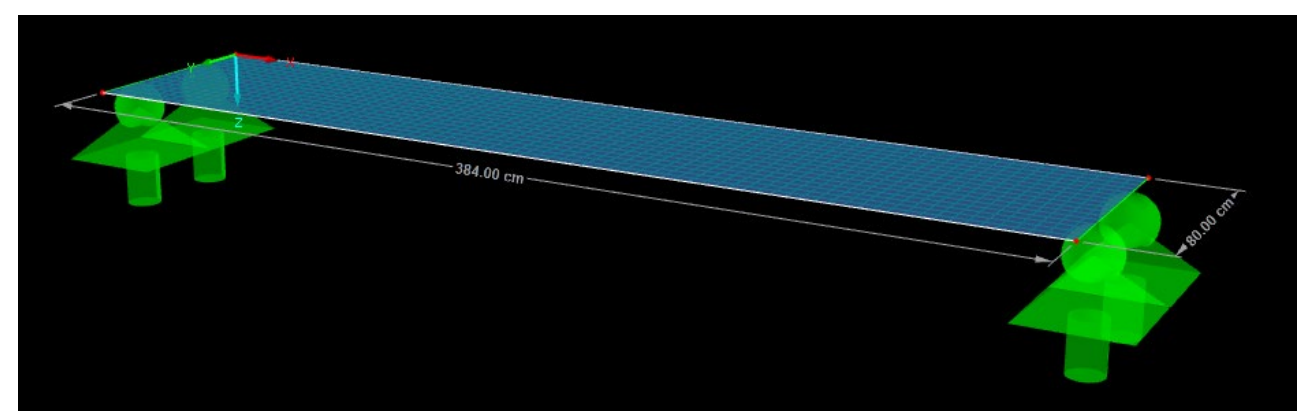

Figura 7. Geometria da placa desenvolvida no modelo numerico

As análises dinâmicas foram realizadas no módulo adicional RF-/DYNAM Pro, sendo que esse modulo fornece uma ferramenta para a análise dinâmica de estruturas mecânicas, sujeitas a excitações externas. Essa extensão é capaz de simular o comportamento de vibração natural de estruturas e estimar os autovalores e autovetores das frequências e modos de vibração, via composição da matriz de massa do sistema estrutural.

A análise do histórico de tempo é efetuada com a análise modal ou com o solucionador implícito de Newmark.

\subsubsection{Elementos de Malha RFEM}

No programa numérico, a análise de estruturas de placa é realizada empregando-se elementos finitos do tipo Shell, consoantes com a teoria de placas de Mindlin-Reissner. Para se garantir um acoplamento direto com elementos de membrana, é escolhida uma abordagem quadrada no plano de casca $\left(\mathrm{u}_{\mathrm{x}}, \mathrm{u}_{\mathrm{y}}\right)$. Ao se eliminar os nós intermediários, um elemento de quatro nós é criado, com um grau adicional de liberdade $\phi_{z}$. Isso permite que os elementos de placa sejam acoplados diretamente aos elementos de viga. Com base em uma interpolação mista de deformações, rotações e distorções, também são aplicados os elementos conhecidos por MITC4 (Interpolação Mista de Componentes Tensoriais), conforme apresentado Figura 8.

A verificação do tamanho e quantidade de elementos finitos que deveriam ser empregados para a análise numérica foi realizada com o Grid Convergence Index (GCI), seguindo o roteiro de cálculo apresentado no item 3.5.1. ver (Vilela, 2020).

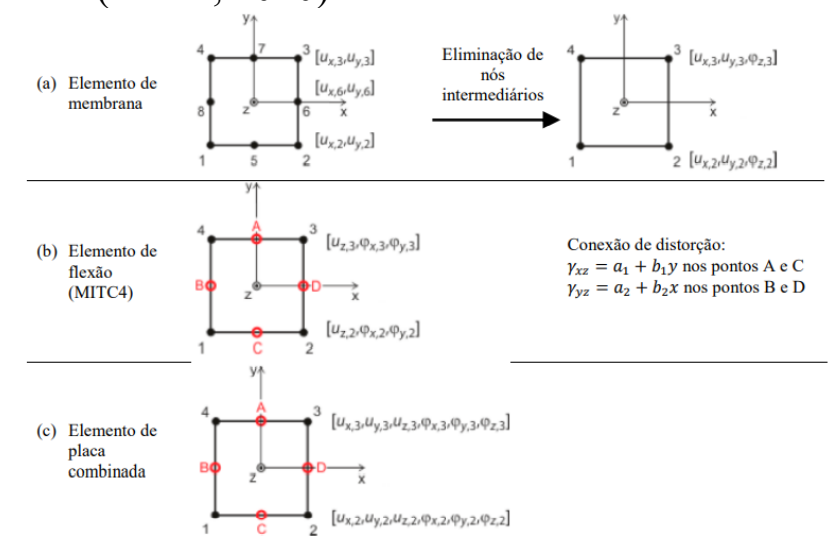

Figura 8. Elementos quadrangulares do RFEM 


\section{RESULTADOS}

Com os resultados de análise numérica pelo método dos elementos finitos com auxílio do software comercial ANSYS, foi possível simular o comportamento da vibração natural da estrutura e estimar as frequências naturais, e os oito primeiros modos de vibração, via composição da matriz de massa do sistema. Os resultados são apresentados na Figura 9.

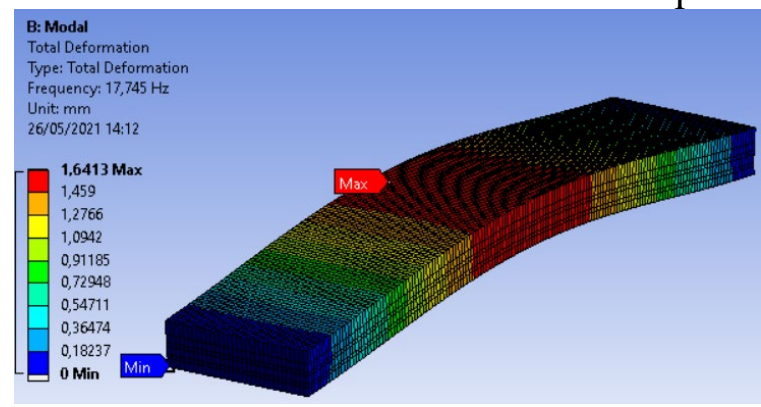

(a) Primeiro modo

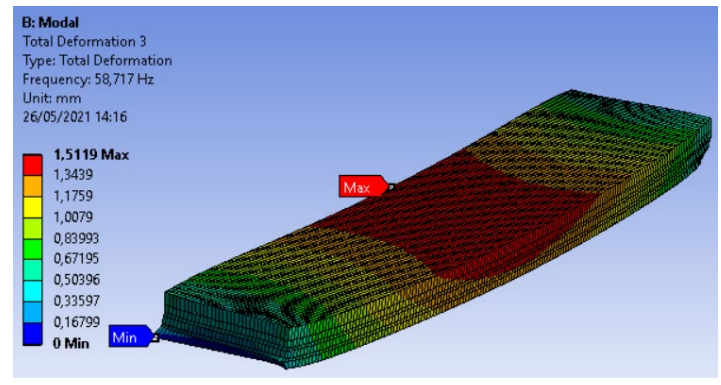

(c) Terceiro modo

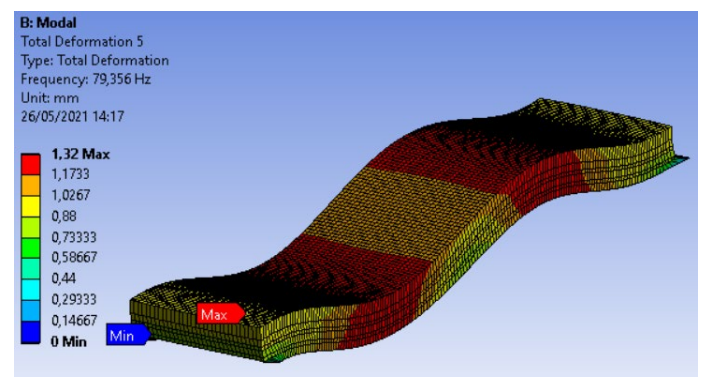

(e) Quinto modo

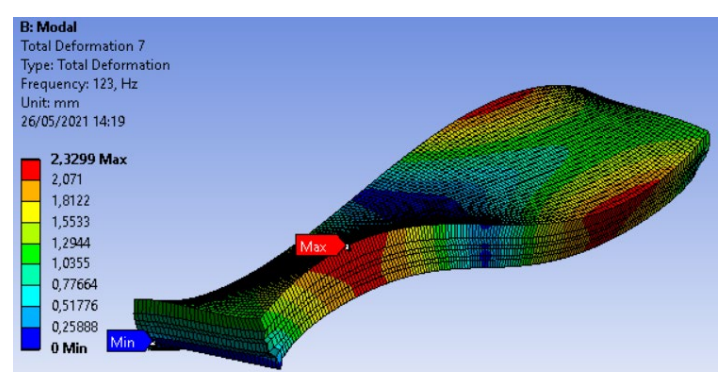

(g) Setimo modo

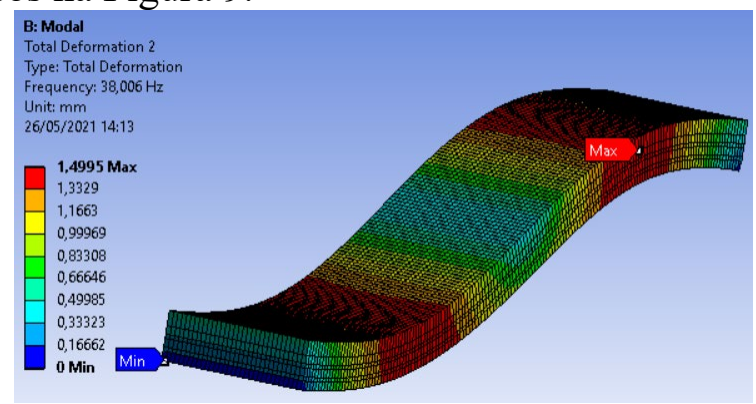

(b) Segundo modo

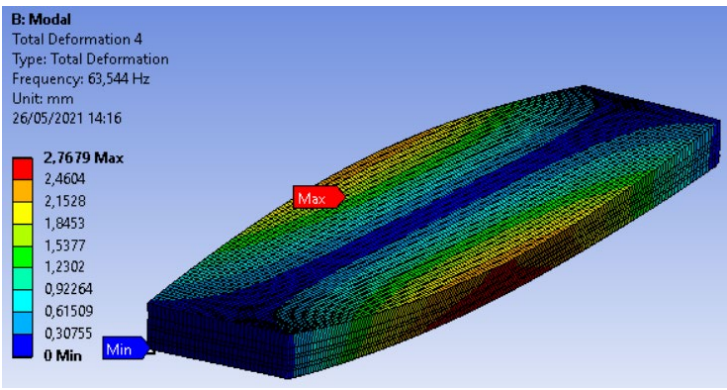

(d) Quarto modo

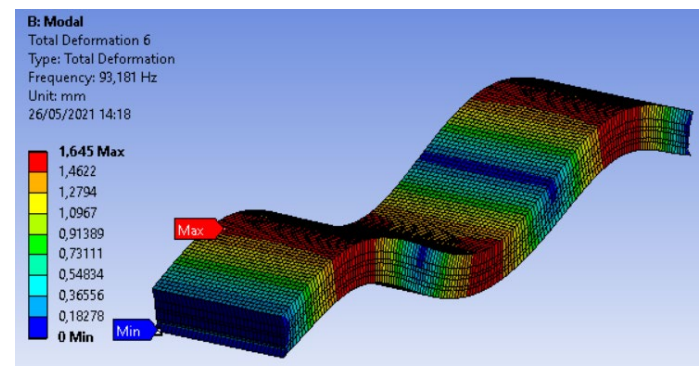

(f) Sexto modo.

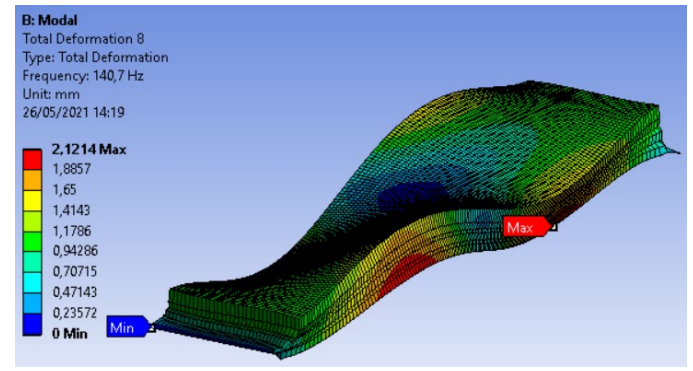

(h) Oitavo modo

Figura 9. Formas de modos de vibração das simulações numéricas ANSYS. 
Considerando-se os resultados obtidos, observou-se que o primeiro modo de vibração flexional de $17,45 \mathrm{~Hz}$, sendo o mais importante deles, teve uma participação de modal nas direções "x", "y", e " $z$ " da ordem de 0,90, 0,89 e 0,97 respectivamente, valores esses, na qual atua a aceleração da estrutura na direção de análise. São aceitáveis para análise dinâmica linear valores nos quais a massa acumulativa seja de pelo menos $80 \%$ na direção predominante da vibração de excitação em que foi analisada.

Na Tabela 5 são apresentados os resultados do processamento númerico do modelo estrutral ,para os oitos modos de vibração elaborada no ANSYS.

Tabela 5. Frequências naturais e seus respectivos modos de vibração ANSYS

\begin{tabular}{|l|c|c|c|}
\hline Modo & $\begin{array}{c}\text { Frequência angular } \\
\boldsymbol{\omega}\left[\frac{\boldsymbol{r a d}}{\boldsymbol{s}}\right]\end{array}$ & $\begin{array}{c}\text { Frequência natural } \\
\mathbf{F}[\mathbf{H z}]\end{array}$ & $\begin{array}{c}\text { Período natural } \\
\mathbf{T}[\mathbf{s}]\end{array}$ \\
\hline $1^{\circ}$ & 111,49 & 17,74 & 0,0563 \\
\hline $2^{\circ}$ & 269,54 & 38,00 & 0,0263 \\
\hline $3^{\circ}$ & 369,92 & 58,71 & 0,017 \\
\hline $4^{\circ}$ & 399,26 & 63,54 & 0,0157 \\
\hline $5^{\circ}$ & 498,62 & 79,35 & 0,01260 \\
\hline $6^{\circ}$ & 587,21 & 93,18 & 0,0107 \\
\hline $7^{\circ}$ & 772,83 & 123,00 & 0,00813 \\
\hline $8^{\circ}$ & 884,08 & 140,07 & 0,007107 \\
\hline
\end{tabular}

Na Tabela 5, é possível observar que as simulações com o peso específico estabelecido conforme as classes NBR 7190 (ABNT ,1997), geraram frequências naturais maiores que $8 \mathrm{~Hz}$. Conforme se aumenta a ordem do modo de vibração é identificada uma ampliação da diferença entre as frequências naturais. Isso demonstra, portanto, que a simulação do primeiro modo de vibração seria a mais crítica, e quando se encontra frequências naturais abaixo de $8 \mathrm{~Hz}$, um estudo mais criterioso das propriedades dinâmicas deve ser efetuado. Contudo se exige que as demais frequências naturais sejam avaliadas, a fim de se dimensionar em intervalos aceitáveis que não coincidam com as probabilidades de ocorrência de ressonância.

Os resultados de análise numérica pelo método dos elementos finitos, com auxílio do software comercial RFEM estão apresentados na Figura 10. 


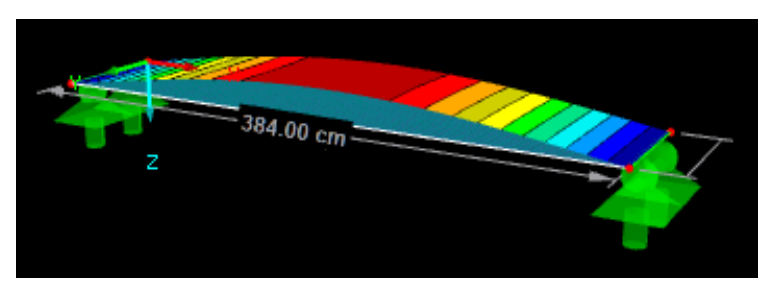

(a) Primeiro modo

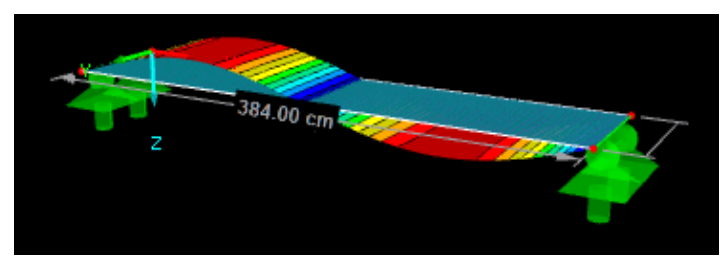

(c) terceiro modo

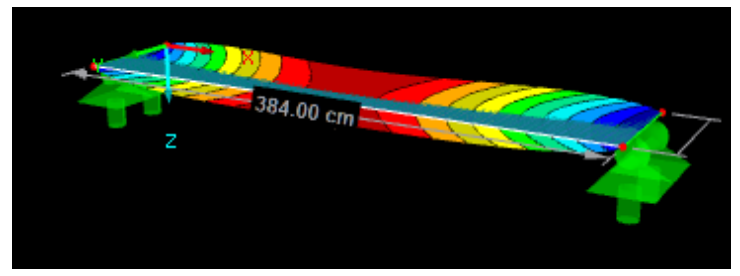

(e) Quinto modo

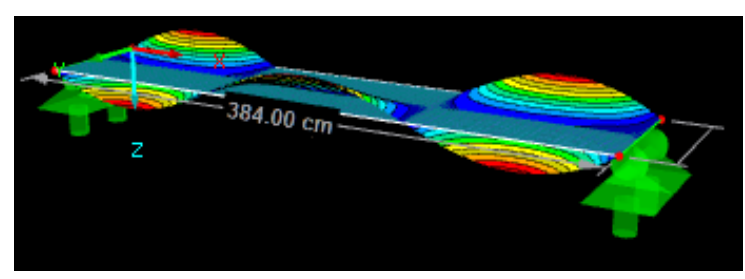

(g) Setimo modo

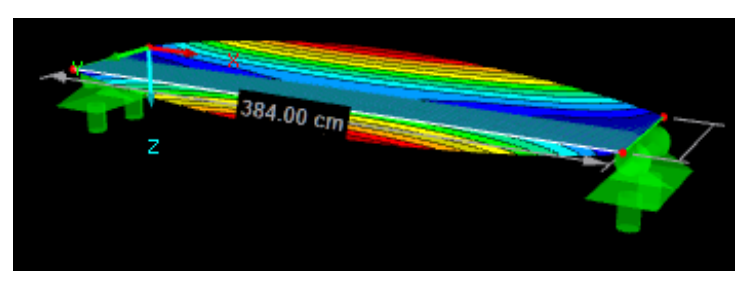

(b) Segundo modo

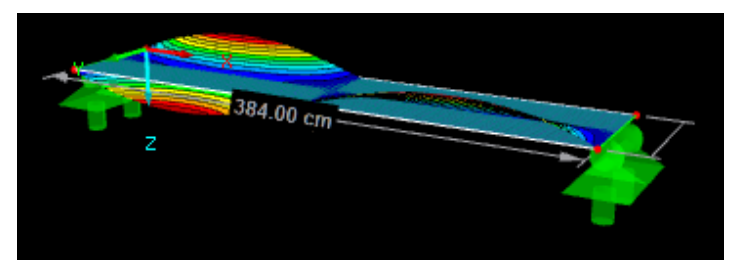

(d) Quarto modo

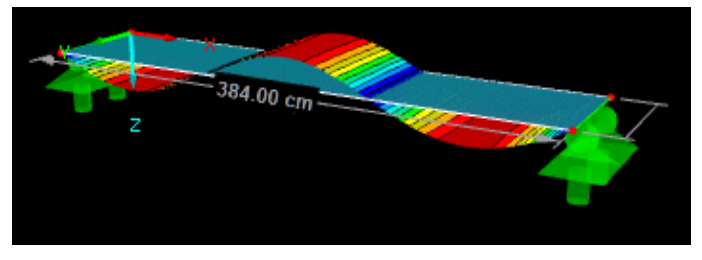

(f) Sexto modo

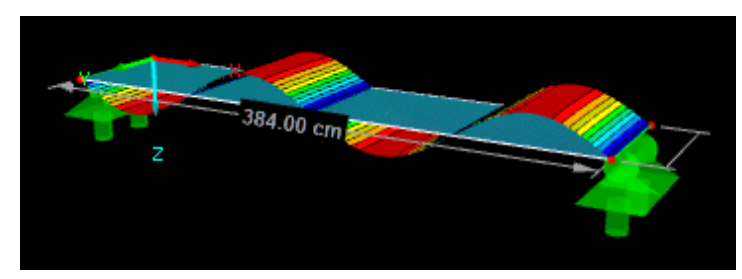

(h) Oitavo Modo

Figura 10. Formas de modos de vibração das simulações numéricas RFEM

A Tabela 6, mostra os resultados do processamento númerico do modelo estrutral, para os oitos modos de vibração Elaborada no RFEM.

Tabela 6. Frequências naturais e seus respectivos modos de vibração RFEM

\begin{tabular}{|l|c|c|c|}
\hline Modo & $\begin{array}{c}\text { Frequência angular } \\
\boldsymbol{\omega}\left[\frac{\boldsymbol{r a d}}{\boldsymbol{s}}\right]\end{array}$ & $\begin{array}{c}\text { Frequência natural } \\
\mathbf{F}[\mathbf{H z}]\end{array}$ & $\begin{array}{c}\text { Período natural } \\
\mathbf{T}[\mathbf{s}]\end{array}$ \\
\hline $1^{\circ}$ & 112,35 & 17,88 & 0,056 \\
\hline $2^{\circ}$ & 267,82 & 42,63 & 0,023 \\
\hline $3^{\circ}$ & 352,79 & 56,149 & 0,018 \\
\hline $4^{\circ}$ & 552,86 & 87,99 & 0,011 \\
\hline $5^{\circ}$ & 568,29 & 90,44 & 0,011 \\
\hline $6^{\circ}$ & 644,13 & 102,52 & 0,010 \\
\hline $7^{\circ}$ & 845,80 & 134,613 & 0,007 \\
\hline $8^{\circ}$ & 936,83 & 149,103 & 0,007 \\
\hline
\end{tabular}


Na Figura 11 mostra os modos de vibração da estrutura sem a contribuição de concreto

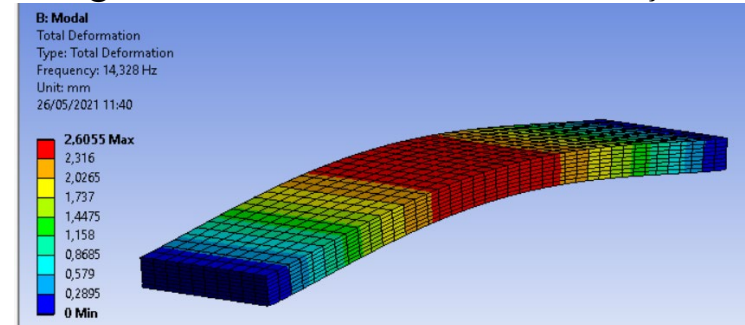

(a) Primeiro modo

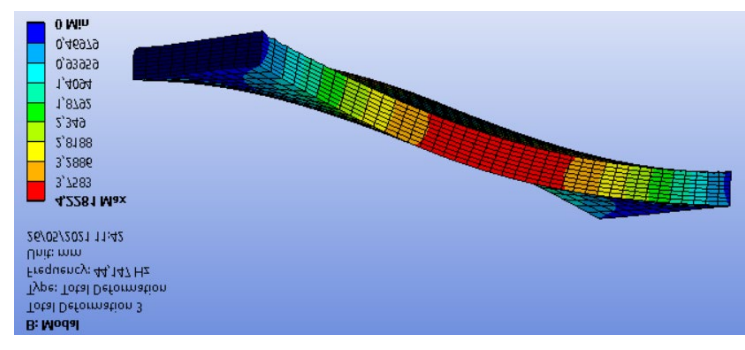

(c) terceiro modo

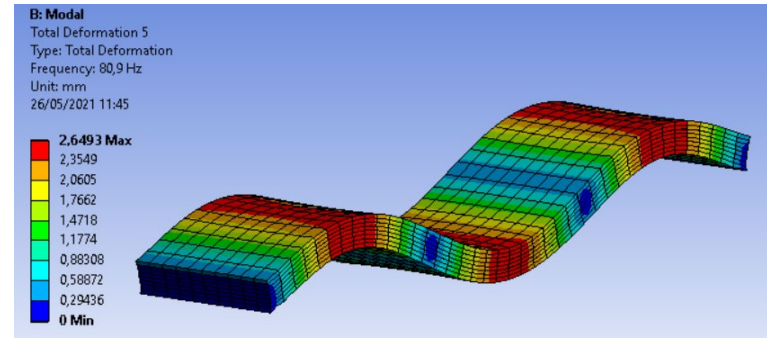

(e) Quinto modo

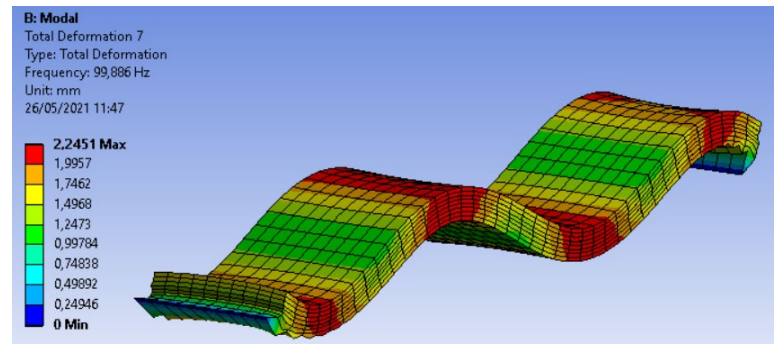

(g) Setimo modo

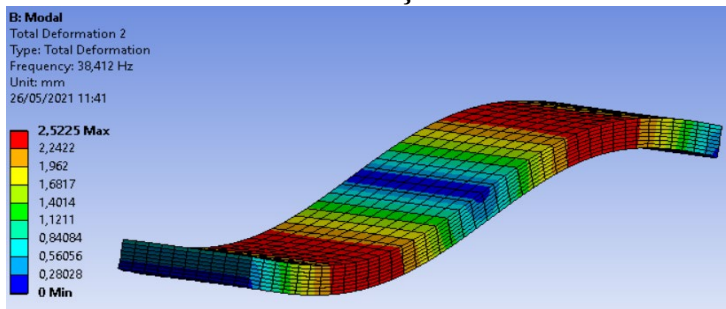

(b) Segundo modo

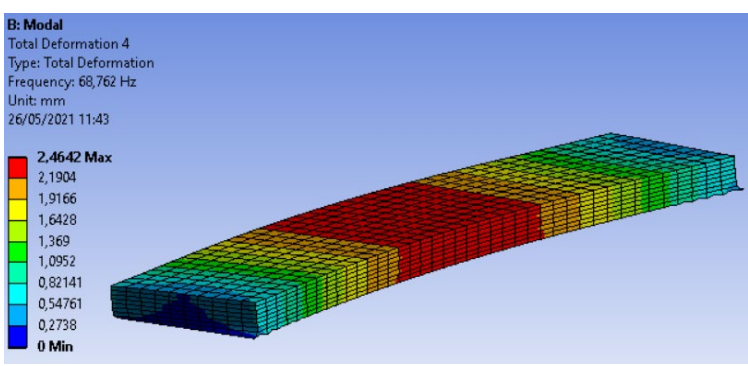

(d) Quarto modo

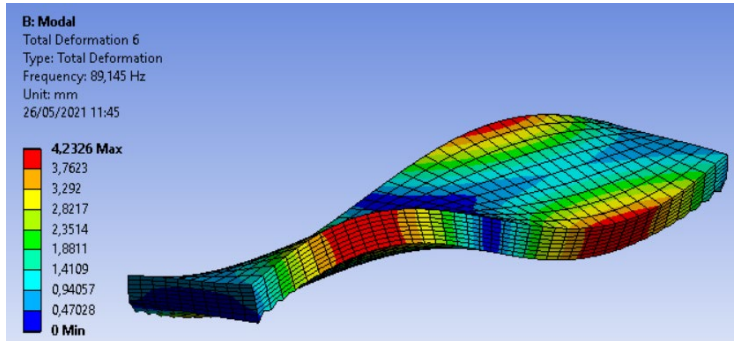

(f) Sexto modo

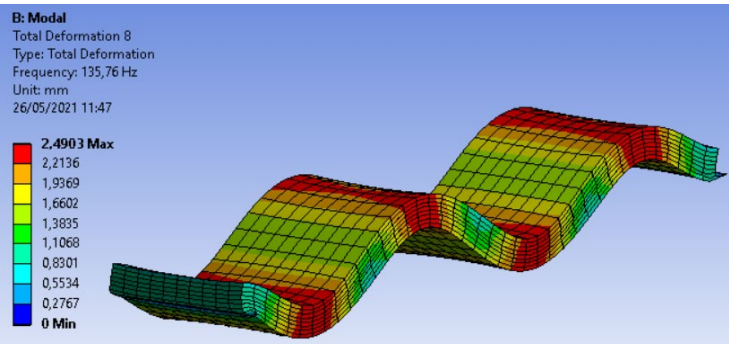

(h) Oitavo Modo

Figura 11. Modos de Vibração sem a contribuição do concreto 
Tabela 7. Frequências naturais e seus respectivos modos de vibração ANSYS sem a contribuiçãoda parcela do elemento concreto

\begin{tabular}{|l|c|c|c|}
\hline Modo & $\begin{array}{c}\text { Frequência angular } \\
\boldsymbol{\omega}\left[\frac{\boldsymbol{r a d}}{\boldsymbol{s}}\right]\end{array}$ & $\begin{array}{c}\text { Frequência natural } \\
\mathbf{F}[\mathbf{H z}]\end{array}$ & $\begin{array}{c}\text { Período natural } \\
\mathbf{T}[\mathbf{s}]\end{array}$ \\
\hline $1^{\circ}$ & 90,016 & 14,32 & 0,0698 \\
\hline $2^{\circ}$ & 241,66 & 38,41 & 0,0260 \\
\hline $3^{\circ}$ & 277,34 & 44,14 & 0,022655 \\
\hline $4^{\circ}$ & 432,04 & 68,76 & 0,014543 \\
\hline $5^{\circ}$ & 508,307 & 80,90 & 0,012361 \\
\hline $6^{\circ}$ & 560,09 & 89,14 & 0,011218 \\
\hline $7^{\circ}$ & 627,56 & 99,88 & 0,010012 \\
\hline $8^{\circ}$ & 852,99 & 135,76 & 0,007366 \\
\hline
\end{tabular}

Na Tabela 7, é possível observar que as simulações com o peso específico estabelecido conforme as classes NBR 7190 (ABNT,1997), foram adotadas as mesmas propriedades mecânicas utilizadas nas Tabela 3 e Tabela 4 , mas sem a parcela de $7 \mathrm{~cm}$ de concreto, a primeira frequência natural de vibração foi de $14,32 \mathrm{~Hz}$, com $3,5 \mathrm{~Hz}$ inferior, se comparado com a estrutura utilizando-se o concreto. Contudo, as frequências naturais não tiveram modos de vibração inferiores a $8 \mathrm{~Hz}$, sendo seu primeiro modo limitante, flexional de $14,32 \mathrm{~Hz}$ e o terceiro modo torcional de $44,14 \mathrm{~Hz}$.

\section{CONCLUSÃO}

Com este trabalho, cujo objetivo foi analisar o comportamento dinâmico de peças estruturais em CLT-CONCRETO a partir de resultados numéricos de elementos finitos, utilizando-se os softwares RFEM e ANSYS, pode-se elencar as seguintes conclusões:

-Os dois modelos numéricos confeccionados no ANSYS e no RFEM com as mesmas propriedades mecânicas e geometrias iguais, com equivalentes condições de contorno, mostraram uma diferença mínima em termos de frequência natural.

-Considerando-se o presente estudo foi obtida uma redução no pico da amplitude do modo de vibrar relacionado à primeira frequência natural de $17,88 \mathrm{~Hz}$, na estrutura de CLT-CONCRETO de $0,8 \%$ se comparada com a mesma estrutura, mas sem a contribuição da parcela de concreto.

-Verifica-se que a parcela de concreto tem uma contribuição significativa para o aumento da rigidez na estrutura, contudo há um acréscimo de peso na peça composta. Caso se aumente a resistência do concreto, pode-se reduzir a sua espessura, e desse modo, será possível melhorar ainda mais os resultados. Entretanto pode-se realizar uma análise na geometria para limitar o máximo vão da estrutura, não somente pela limitação da flecha, mas como também pelos critérios de análise dinâmica.

Nesse contexto, se o objetivo for o de eliminar um determinado modo de vibrar, que coincide com o modo da frequência natural da estrutura, essa frequência de excitação gera o fenômeno conhecido como ressonância, assim, o sistema físico passa a vibrar com amplitudes cada vez maiores, chegando ao ponto de a estrutura sofrer sérios danos, ou até mesmo colapsar. Para se evitar essa aproximação, torna-se viável a utilização de camadas de concreto para conseguir o objetivo proposto. 


\section{AGRADECIMENTOS}

Os autores agradecem a Crosslam, empresa que fornece os materiais para análise e aos técnicos do Laboratório de Estruturas da FEC-Unicamp na realização dos ensaios experimentais.

\section{REFERENCIAS}

ALOISIO, A. et al. (2020) "Dynamic identification and model updating of an eight-storey CLT building. Engineering Structures", v. 213, p. 593.

ANSYS User 's Manual. Theory manual. (2009). "revision 12.02"

Associação Brasileira de Normas Técnicas. (1997). NBR 7190: Projeto de estruturas de madeira. Rio de Janeiro.

BACHMANN, H.; AMMANN, W. (1987). "Vibrations in Structures: Induced by Man and Machies." IABSE.

CLOUSTON, P.; SCHREYER, A. C. (2006) “Wood-Concrete Composites: A Structurally Efficient Material Option. Civil Engineering Practice, Journal of the Boston Society of Civil Engineers" (BSCE), v. 21, p. 5-22.

DE SILVA, C. W. (2000) "Vibration: fundamentals and practice". Boca Raton, FL: CRC.

DLUBAL SOFTWARE GMBH. REFEM 5 (2013). "Program Description. Tiefenbach: Dlubal" Software GmbH.

JIANG, Y.; CROCETTI, R. (2019), “CLT-concrete composite floors with notched shear connectors. Construction and Building Materials", v. 195, p. 127-139.

LIMA, S.; HAMPSHIRE DE CARVALHO SANTOS, S. (2008) “Análise Dinâmica de Estruturas".

PEREIRA, M. C.; CALIL, C. (2019), "Strength and Stiffness of Cross Laminated Timber (CLT) panels produced with Pinus and Eucalyptus: experimental and analytical comparisons." Matéria (Rio de Janeiro), v. 24, 1

RAO, S. S. (2008), “Vibrações Mecânicas. ”4. ed. SÃO PAULO: Pearson Education.

SUÁREZ-RIESTRA, F. et al. (2019), "Experimental, analytical and numerical vibration analysis of long-span timber-timber composite floors in self-tensioning and non-tensioning configurations. Construction and Building Materials", v. 218, p. 341-350.

VILELA, R. (2020) “DESEMPENHO ESTRUTURAL DE PLACAS DE CROSS LAMINATED TIMBER SUBMETIDAS À FLEXÃO.” CAMPINAS: ESTADUAL DE CAMPINAS (UNICAMP), 120p.

XING, Y.; LIU, B. (2009), "New exact solutions for free vibrations of rectangular thin plates by symplectic dual method. Acta Mechanica Sinica”, v. 25, n. 2, p. 265-270. 